\title{
Dolor crónico posoperatorio: Una revisión necesaria de un problema ignorado
}

\section{Chronic post-surgical pain: A necessary review for a disregarded problem}

\author{
María Francisca Bernucci Pérez MD. ${ }^{1}$, Waldo Andrés Merino Urrutia MSc, MBA. ${ }^{2}$, Marisabel Fernanda Díaz Gil³, \\ Matías Julián Feijoo Palacios ${ }^{3}$, Juan José Orellana Cáceres PhD. ${ }^{4}$, Marco Alejandro Balkenhol Neumann ${ }^{5}$, \\ Orlando Felipe Navarro Véliz ${ }^{5}$, Claudio Iván Cárcamo Fuentes MSc. ${ }^{6}$, David Torres Pérez ${ }^{7}$, Rubén Felipe Carrasco Moyano ${ }^{8}$ \\ 1 Servicio de Anestesiología y Dolor del Hospital de Carabineros, Santiago de Chile. Universidad Mayor. Santiago, Chile. \\ 2 Departamento de Cirugía, Traumatología y Anestesia. Centro de Capacitación Investigación y Gestión en Salud para la Medicina \\ Basada en Evidencias (CIGES), Facultad de Medicina, Universidad de La Frontera. Temuco, Chile. \\ 3 Alumno de Medicina Universidad de La Frontera, Temuco, Chile. \\ 4 Centro de Capacitación Investigación y Gestión en Salud para la Medicina Basada en Evidencias (CIGES), Universidad de La Fron- \\ tera. Temuco. Chile.. \\ 5 Universidad San Sebastián, Hospital Base de Puerto Montt. Puerto Montt, Chile. \\ 6 Enfermero Universitario. Departamento de Enfermería. Facultad de Medicina. Universidad de La Frontera. Temuco, Chile. \\ 7 Universidad de Los Andes. Santiago, Chile. \\ 8 Hospital Hernán Henríquez Aravena. Temuco, Chile.
}

Fecha de recepción: 23 de agosto de 2020 / Fecha de aceptación: 12 de septiembre de 2020

\begin{abstract}
Introduction: Postoperative chronic pain (PCP) is defined as a discomfort that lasts more than expected or beyond 3 months after surgery. The recognition and study of this clinical entity has been gaining interest in the past few years. One of the main reasons is the great impact it can have on the quality of life of patients who suffer from it. Objective: To describe the prevalence, risk factor and impact of PCP on patients subjected to different surgical procedures. Materials and Methods: Search on MEDLINE/Pubmed, using the following terms: "chronic postsurgical pain", "chronic post operative pain", "chronic post operative pain", "chronic post surgical pain". Publications were then sieved using their title and abstract. Results: Fifty-seven articles were analyzed. Ten to fifty percent of patients were reported as suffering PCP, with up to $10 \%$ incidence of severe pain. The main risk factors identified were the use of analgesics, pre-operative pain and type of surgery. Orthopedic surgery was highly associated with moderate to severe PCP within the first year. Twenty-one percent of patients referred PCP affected their quality of life and sleep; ability to carry out physical activity and return to work. Conclusion: The first approach to PCP is getting to know its impact and epidemiology. Surgical treatments can cause PCP, which will affect patient recovery and increase costs. Recognizing those patients at risk and establishing preventive management protocols could improve the quality of life of postsurgical patients.
\end{abstract}

Key words: Chronic pain, postoperative period, chronic postsurgical pain, chronic postoperative pain, chronic post surgical pain and chronic post operative pain.

\section{RESUMEN}

Introducción: El dolor crónico posoperatorio (DCPO) es aquel que aparece tras un procedimiento quirúrgico y se mantiene por más tiempo de lo esperado o más de tres meses luego de la cirugía. Es una entidad de reciente estudio y que tiene un gran impacto en la calidad de vida de los pacientes postoperados. Objetivo: Describir la prevalencia, factores de riesgo y el impacto del DCPO en pacientes sometidos a diferentes procedimientos quirúrgicos. Material y Método: Se realizó una revisión de la literatura utilizando la base de datos MEDLINE/Pubmed. Los términos libres fueron "chronic postsurgical pain", "chronic postoperative pain", "chronic post operative pain", "chronic post surgical pain". Se realizó un cribado según título y resumen. Resultados: Fueron analizados 57 artículos. De 10\%-50\% de los pacientes presentan DCPO y hasta 10\% puede ser severo. El uso de analgésicos, presencia de dolor preoperatorio y especialidad quirúrgica han sido señalados como los principales factores

waldo.merino@ufrontera.cl

https://orcid.org/0000-0002-4956-8444 
de riesgo. La cirugía traumatológica aparece como la más asociada a DCPO moderado y severo a un año. Hasta $21 \%$ de los pacientes refieren que afecta su calidad de vida, el sueño, actividad física y reincorporación laboral. Conclusión: El conocimiento de la epidemiología e impacto del DCPO es un primer acercamiento a este problema. Patologías de resolución quirúrgica pueden evolucionar con DCPO, afectando su recuperación y aumentando los costos que se desea contener. El reconocimiento de la población de riesgo y la instauración de protocolos preventivos pudiera mejorar la calidad de vida de los pacientes postoperados.

Palabras clave: Dolor crónico, postoperatorio, dolor crónico posquirúrgico, dolor crónico posoperatorio, dolor crónico posquirúrgico, y dolor crónico posoperatorio.

\section{Introducción}

E dolor crónico posoperatorio (DCPO) es aquel que aparece luego de someterse a un procedimiento quirúrgico y se mantiene por más tiempo de lo esperado o más allá de tres meses luego de la cirugía. La incidencia reportada varía en función de la metodología de los estudios y la definición utilizada. La definición aceptada por la IASP (Asociación Internacional para el Estudio del Dolor) es[1]:

1. Aquel dolor que se prolonga más allá de dos a seis meses después de una cirugía.

2. El dolor no estaba presente previo a la cirugía o si lo estaba, ha cambiado en sus características y/o intensidad respecto al dolor preoperatorio.

3. El dolor se localiza en el sitio quirúrgico o zonas de dolor referido acordes.

4. Se ha descartado otras causas de dolor.

El DCPO es una entidad que recién empieza a ser discutida en la literatura científica a fines de los años '90. Su definición precisa aún es tema de debate. Existe poca información de su perfil epidemiológico a nivel mundial, pese a que tiene un impacto tanto para el paciente como para los sistemas de salud y la economía.

El propósito de nuestro estudio es, por medio de una búsqueda bibliográfica amplia, conocer la prevalencia y factores de riesgo determinantes de dolor crónico postquirúrgicos, en pacientes adultos, descritos en la literatura para alcanzar una apreciación general acerca de la magnitud de esta complicación en los pacientes.

\section{Material y Método}

Llevamos a cabo una revisión de la literatura mediante el uso de la metodología PICOR. Se utilizó como fuente de búsqueda bibliográfica la base de datos MEDLINE/PubMed. Utilizamos los términos MeSH "chronic pain" y "postoperative period" sin resultados. Luego la búsqueda fue ampliada, de acuerdo a publicaciones conocidas por los autores, con los siguientes términos libres: "chronic postsurgical pain", "chronic postoperative pain", "chronic post operative pain", "chronic post surgical pain". Realizamos la siguiente estrategia de búsqueda: Search ((()("chronic postsurgical pain") OR "chronic postoperative pain") $O R$ "chronic post operative pain") $O R$ "chronic post surgical pain")) AND review[Title/Abstract]. No se aplicaron filtros de idiomas, si para estudios en humanos, adultos, en los últimos cinco años. La búsqueda fue realizada por cada autor. Se realizó un cruzamiento de las publicaciones y fueron selec- cionadas aquellas que contaron con tres o más coincidencias. Luego, se agregó por consenso aquellas en la que había menos de tres coincidencias. Las discrepancias fueron resueltas por consenso. De esta manera el número total de publicaciones seleccionadas fue de 113 artículos. A éstos se les realizó un cribado por títulos y resumen y se añadió aquellos trabajos conocidos por los autores, obteniéndose 68 artículos. Finalmente, por consenso, la selección fue limitada a 50 artículos (Figura 1).

\section{Resultados}

De los artículos seleccionados, 45 fueron estudios observacionales (estudios de prevalencia, de factores de riesgo (FR) e impacto en la calidad de vida de los pacientes), un artículo de revisión, dos ensayos clínicos, una revisión sistemática y un metaanálisis. Los casos clínicos no fueron incluidos.

\section{Incidencia}

La incidencia publicada de DCPO es de 10\% a 50\% y varía en función de la metodología de los estudios y la definición utilizada[2]. De los pacientes afectados, 2 a 10\% pueden presentar dolor severo[3]. El dolor es capaz de impedir que puedan reintegrarse a sus labores habituales y retrasa su reincorporación al trabajo[3],[4].

\section{Factores de riesgo}

Se han identificado FR propios del paciente como son el uso de analgésicos, la presencia de dolor pre y posoperatorio; y factores quirúrgicos, como el tipo de cirugía[3],[5],[6]. Hoofwijk et al. describieron la prevalencia y FR de DCPO en pacientes ambulatorios. De un universo de 1.396 pacientes, el 15,3\% experimentó DCPO moderado a severo y 2,3\% lo describió como severo. Los principales predictores de DCPO fueron la especialidad quirúrgica, dolor agudo prequirúrgico (DAPQ), uso de analgésicos preoperatorios, miedo a la cirugía y dolor agudo postquirúrgico. El DAPQ triplicó las posibilidades de desarrollar DCPO[7]. La cirugía traumatológica presentó mayor asociación con dolor moderado y severo al cabo de un año.

Revisiones sistemáticas describen que el dolor catastrófico (magnificación de la gravedad o sensación de impotencia en contexto del dolor) estaría asociado a DCPO. Este es mayor en pacientes sometidos a cirugía musculoesquelética y es el mayor predictor de DCPO en artroplastia total de rodilla. La ansiedad, depresión, estrés y el retorno tardío al trabajo como FR presentan resultados inconsistentes[8].

Se ha intentado predecir la aparición de DCPO, para evitar- 


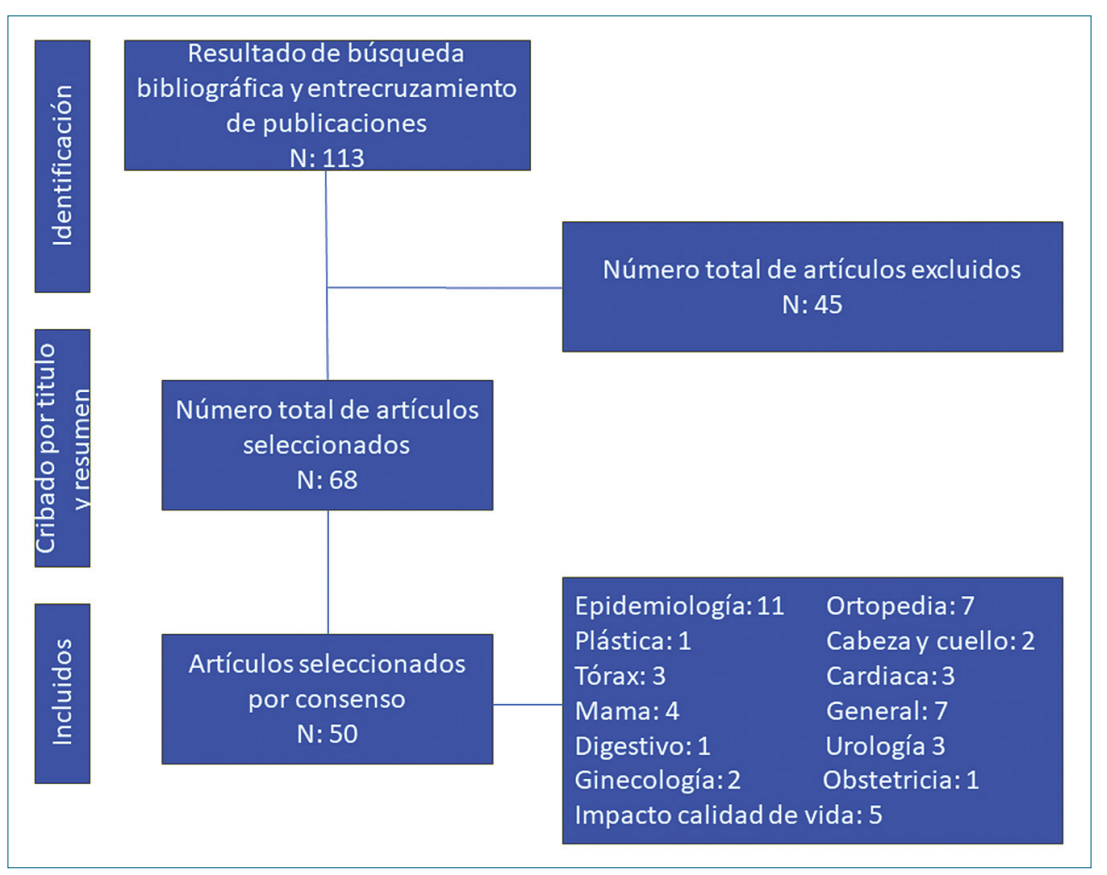

Figura 1. Flujograma de selección de artículos.

lo o al menos manejarlo precozmente. Chatel et al., describieron factores modificables y no modificables que inciden en la aparición de dolor. Con esos datos, plantearon explícitamente un plan de intervención en el perioperatorio, como parte de las estrategias para evitar la aparición de dolor persistente[9]. Althaus et al., han publicado el desarrollo de un índice de riesgo para predecir la aparición de DPCO[10]. Sin embargo, su índice está basado en FR estudiados sólo hasta el 2007, por lo que algunos de ellos ya han sido descartados por estudios posteriores. Además, con sólo 150 pacientes, su potencia estadística es débil. Existen otros intentos por predecir DCPO, pero sólo para cirugías acotadas[11]. A la fecha, no se ha validado una escala de riesgo que prediga la aparición de este fenómeno.

Dado que la especialidad quirúrgica es un FR importante, a continuación describiremos las más relevantes mencionadas en la literatura. Esta información se encuentra resumida en la Tabla 1.

\section{Cirugías ortopédicas}

En este grupo el DCPO está reportado entre 15\%-65\%. Los principales FR identificados son la técnica quirúrgica, la existencia de DAPQ, uso previo de opioides, tabaquismo, trastornos del ánimo y dolor en el posoperatorio inmediato[12]-[18]. Zaslansky et al., en un estudio de cohorte retrospectivo derivado de la base de datos PAIN OUT para el registro del dolor en cirugía ortopédica, consignó FR distintos a los habitualmente reportados, relacionando la persistencia del dolor con un "excesivo" aparato clínico para su seguimiento, las instituciones docentes e incluso el tipo de seguro de salud[12].

La cirugía de trauma ortopédico se ha asociado con la incidencia más alta de DCPO (65\%). Los FR identificados corresponden a sexo femenino; antecedente de cirugía por trauma ortopédico previo e intensidad de dolor prequirúrgico. Además, la presencia de DCPO interfirió con las actividades de los pa- cientes y se asoció a discapacidad, angustia y mayor uso de recursos[15].

La artroplastia total de rodilla es un procedimiento quirúrgico efectivo para mejorar la función y reducir el dolor articular de pacientes en etapa final de artrosis. Sin embargo, hasta 34\% de los pacientes persisten con DCPO moderado a severo después de tres meses de la cirugía[16]. Rice et al., realizaron un estudio de cohorte prospectivo con una muestra de 300 pacientes, concluyendo que a pesar de la efectividad en la reducción del dolor en la mayoría de los pacientes, entre 16\% y $21 \%$ presentó DCPO moderado a severo a seis y 12 meses. Dentro de los FR más importantes de DCPO se encuentra dolor prequirúrgico, la intensidad del DAPQ, rasgo de personalidad ansioso y dolor esperado posterior a cirugía[14],[17].

La artroscopia de rodilla es un procedimiento frecuente, siendo una de sus principales indicaciones el dolor crónico de rodilla. Paradojalmente, el dolor constituye una de las complicaciones del procedimiento. Hoofwijk et al., en una cohorte prospectiva, evaluaron la prevalencia y FR de DCPO en artroscopia de rodilla. El 32,7\% de los pacientes presentaron dolor moderado a severo a los 18 meses. Los FR identificados fueron la presencia de dolor preoperatorio y el uso preoperatorio de analgésicos[18].

\section{Cirugía plástica}

En una serie retrospectiva Chatel et al., revisaron la cirugía de contorno corporal y DCPO. Como parte de la evaluación, se aplicaron encuestas clínicas orientadas a caracterizar el dolor y un cuestionario sobre impacto social y psicológico del dolor. La prevalencia descrita fue de $21 \%$, con un importante componente neuropático(70\%). Fueron identificados FR tales como el DAPQ, el tipo de seguro de salud, antecedentes de cirugía bariátrica, miedo al procedimiento y el haber sufrido complicaciones. No hubo asociación con la edad, sexo, tabaquismo e IMC alto[9]. 
Artículo de Revisión

\begin{tabular}{|c|c|c|c|}
\hline Cirugía & Incidencia & Factores de riesgo & Referencia \\
\hline Trauma ortopédico & $65 \%$ & $\begin{array}{l}\text { - Sexo femenino } \\
\text { - Antecedente cirugía de trauma ortopédico previo } \\
\text { - Dolor agudo prequirúrgico (DAPQ) }\end{array}$ & 15 \\
\hline Artroplastía rodilla & $34 \%$ & $\begin{array}{l}\text { - Intensida DAPQ } \\
\text { - Personalida ansiosa } \\
\text { - Expectativa de dolor pos-quirúrgico }\end{array}$ & $14,16,17$ \\
\hline Artroscopia rodilla & $32,7 \%$ & $\begin{array}{l}\text { - DAPQ } \\
\text { - Uso preoperatorio de analgesicos }\end{array}$ & 18 \\
\hline Cirugía plástica contorno corporal & $21 \%$ & $\begin{array}{l}\text { - DAPQ } \\
\text { - Antecedente de cirugía bariátrica } \\
\text { - Miedo al procedimiento } \\
\text { - Antecedente de complicaciones } \\
\text { - Tipo de seguro de salud }\end{array}$ & 9 \\
\hline Tiroidectomía & $9 \%-12 \%$ & - Técnica anestésica (bloqueo cervical superficial) & 19 \\
\hline $\begin{array}{l}\text { Timpanoplastía/ } \\
\text { Timpano-mastoidectomía }\end{array}$ & $36 \%-39 \%$ & $\begin{array}{l}\text { - Migraña } \\
\text { - Patología cervical } \\
\text { - DAPQ }\end{array}$ & 20 \\
\hline Mastoidectomía & $32,3 \%$ & $\begin{array}{l}\text { - DAPQ } \\
\text { - > } 30 \text { años }\end{array}$ & 21 \\
\hline Cirugía Tórax & $25 \%-33 \%$ & $\begin{array}{l}\text { - DAPQ } \\
\text { - Sexo femenino }\end{array}$ & 22,23 \\
\hline Cirugía cardíaca & $9,5 \%-56 \%$ & $\begin{array}{l}\text { - Uso de arteria torácica interna para by-pass } \\
\text { - Antecedente uso de antidepresivos } \\
\text { - Hipotiroidismo } \\
\text { - Complicaciones herida operatoria }\end{array}$ & 27 \\
\hline Cirugía mama & $20 \%-60 \%$ & $\begin{array}{l}\text { - Pacientes jóvenes (<66 años) } \\
\text { - Mastectomía (vs cirugía conservadora) } \\
\text { - Resección ganglios axilares } \\
\text { - DAPQ } \\
\text { - Dolor neuropáticos post-operatorio }\end{array}$ & $28,29,30$ \\
\hline Hernia inguinal & $10 \%-53 \%$ & $\begin{array}{l}\text { - Edad* } \\
\text { - Tipo de malla } \\
\text { - Fijación de malla* }\end{array}$ & $\begin{array}{l}32,35 \\
37 \\
36,37\end{array}$ \\
\hline Cirugía colorrectal & $17 \%$ & $\begin{array}{l}\text { - DAPQ } \\
\text { - Enfermedad inflamatoria intestinal } \\
\text { - Filtración anastomosis }\end{array}$ & 38 \\
\hline Nefrectomía & $4 \%-16 \%$ & $\begin{array}{l}\text { - DAPQ } \\
\text { - Antecedente cirugía abdominal } \\
\text { - Lumbago preexistente }\end{array}$ & 40,41 \\
\hline Histerectomía & $5 \%-50 \%$ & $\begin{array}{l}\text { - DAPQ } \\
\text { - Ansiedad-depresión } \\
\text { - Dolor agudo posquirúrgico } \\
\text { - Insatisfacción }\end{array}$ & 42 \\
\hline Esterilización histeroscópica & $4,2 \%$ & $\begin{array}{l}\text { - Antecedente dolor pélvico crónico } \\
\text { - Dolor lumbar crónico } \\
\text { - Cefalea } \\
\text { - Fibromialgia }\end{array}$ & 43 \\
\hline Cesárea & $6,8 \%-79 \%$ & $\begin{array}{l}\text { - Cesárea previa } \\
\text { - > Consumo analgésicos } \\
\text { - Depresión preoperatoria } \\
\text { - Duración de cirugía } \\
\text { - DAPQ }\end{array}$ & 44 \\
\hline
\end{tabular}

$\mathrm{DAPQ}=$ Dolor agudo pre-quirúrgico; *Factor de riesgo controversial en la literatura. 


\section{Tiroides-Cabeza y cuello}

Wattier et al., estudiaron 304 pacientes luego de una tiroidectomía, encontrando una incidencia de DCPO de $12 \%$ a los tres meses y $9 \%$ a los seis meses. El análisis multivariado demostró que el tipo de anestesia modifica el riesgo. En aquellos pacientes que no recibieron bloqueo cervical superficial el DCPO fue tres veces más frecuente[19]. En 150 pacientes sometidos a timpanoplastia o timpanomastoidectomía, Güven et al., describió que la mastoidectomía no posee un riesgo adicional de DCPO, excepto en pacientes con migraña, patología cervical y DAPQ[20]. Shadman et al.[21], estudiaron la frecuencia de DCPO en 155 pacientes sometidos a mastoidectomía, (simple, radical y radical modificada), observándose en 50 pacientes $(32,3 \%)$. El dolor agudo severo poscirugía incrementó la incidencia de DCPO 17 veces y los mayores de 31 años tuvieron seis veces mayor probabilidad de padecerlo.

\section{Tórax}

La toracotomía y videotoracoscopía son algunos de los procedimientos con mayor riesgo de DCPO. Diferentes publicaciones describen una prevalencia de DCPO a seis meses de $33 \%$ y de $25 \%$ para toracotomía y videotoracoscopía. Estas diferencias no alcanzaron una significación estadística, tampoco respecto del dolor agudo posquirúrgico, lo cual es algo inesperado considerando las importantes diferencias quirúrgicas del procedimiento[22]. Aspectos psicosociales como depresión, fatiga, ansiedad y disturbios del sueño, entre otros, no presentaron un rol predictivo.

Se ha reconocido como causa de DCPO el pobre manejo del dolor postoperatorio inmediato. Niraj et al., describieron que de los pacientes que presentaron cinco o más episodios de dolor dentro de las $72 \mathrm{~h}$ posoperatorias, el $82 \%$ tenía DCPO a los seis meses. De esta manera, nuevamente el DAPQ fue predictor de $\mathrm{DCPO}[23]$.

Florelli et al., en 200 pacientes, describió que en quienes se realizó una resección pulmonar mediante minitoracotomía o toracoscopia la incidencia de DCPO fue del 35\%, de los cuales el 31,5\% presentaron dolor neuropático. Solo el 10\% de los pacientes con DCPO informaron dolor crónico intenso. Según el análisis univariante, la DCPO se asoció a dolor posoperatorio agudo moderado y severo $(p<0,001)$ y género femenino $(p<$ $0,044)$. Según el análisis multivariable, los factores de riesgo independientes para el desarrollo de DCPO incluyeron la aparición de dolor posoperatorio agudo de moderado a severo (OR $32,61 ; p<0,001)$ y cirugía abierta (OR 6,78; $p<0,001)$. La incidencia de dolor neuropático fue mayor en pacientes femeninas ( $16 \%$ en mujeres y $6 \%$ en hombres; $p<0,040)$. Se registró una disminución significativa en todos los puntajes del dominio de la Encuesta de Salud SF-36 para pacientes con DCPO y dolor neuropático $(p<0,001)[24]$.

\section{Cirugía cardíaca}

En cirugía cardíaca, se reporta una prevalencia de DCPO de $9,5 \%$ a $56 \%$. Su presencia altera y disminuye la calidad de vida de los pacientes[25]. Marcassa et al., estudiaron retrospectivamente 313 pacientes, observando una prevalencia de 35,3\% a los tres meses, $26,8 \%$ al año y de $19,8 \%$ a los tres años ( $p<$
0,05). En el grupo de 3 años, el dolor fue calificado de moderado a severo por un tercio de los pacientes[26]. Costa MA et al., en 453 pacientes, identificaron los siguientes FR para DCPO después de una esternotomía: uso de la arteria torácica interna para bypass, historia de uso de antidepresivos, hipotiroidismo y complicaciones de la herida operatoria[27].

\section{Cirugía mama}

Si bien el dolor agudo asociado a la cirugía de mama se resuelve en la mayoría de los casos, el DCPO afecta a un número importante de pacientes, con una incidencia variable entre 20\%-60\%, impactando de forma negativa en la calidad de vida y causando una reducción de la funcionalidad física. El DCPO ha sido descrito en una amplia variedad de procedimientos quirúrgicos que incluyen aumento mamario, mamoplastia de reducción, mastectomía y mastectomía con reconstrucción.

Las características de las paciente asociadas al desarrollo de dolor crónico después de una cirugía estética de mama incluye una edad más joven, IMC más grande, menor altura, hiperestesia postoperatoria y depresión basal elevada y ansiedad[28],[29].

Andersen et al., reconocen FR asociados a DCPO en cirugía de mama, como son: la edad $<65$ años ( $p<0,02$ ), la cirugía conservadora vs mastectomía $(p<0,006)$, la disección de ganglio linfático axilar con preservación del nervio intercostal $(p<0,0005)$, la presencia de dolor agudo posquirúrgico $(p<0,0018)$ y signos de dolor neuropático en la primera semana $(p<0,01)[30]$.

Nishimura et al., plantean la disección de ganglios linfáticos axilares como un FR modificable[29]. García et al., describen $36,5 \%$ de DCPO luego de dos años transcurridos de una mastectomía. EI DCPO dificulta la recuperación, obligando a los pacientes a re-consultar, a requerir medicación crónica y a un aumento de gastos médicos[4].

\section{Cirugía hernia inguinal}

En la reparación de hernia inguinal con malla, el DCPO es considerado como la complicación postquirúrgica más común a largo plazo, con una incidencia entre 10\%-53\%[31]-[33]. En pacientes con reparación uni- o bilateral, el grado en que el dolor puede llegar a afectar el nivel de actividad de los pacientes, es similar entre pacientes con DCPO de uno o ambos lados[34].

Langeveld et al., en un estudio de 669 pacientes con un rango de edad entre 19-89 años, identificó mayor riesgo de DCPO a menor edad: 43\% (18-40 años) vs 29\% (40-60 años) vs 19\% (> 60 años) ( $p<0,001)$ [35]. Esto se contradice a lo estudiado en 334 pacientes por Takata et al., donde los resultados sugieren que no existe asociación significativa entre edad y DCPO.

Takata recomienda que se debiera realizar una identificación rigurosa de los nervios ilioinguinal, iliohipogástrico y la rama genital del nervio genitofemoral, preservando y evitando el posicionamiento de la malla en contacto con éstos, para así reducir el riesgo de DCPO[32].

No se ha demostrado una diferencia significativa de DCPO al comparar la técnica Lichtenstein (10,9\% de DCPO) vs la técnica sin fijación de la malla ONSTEP) (13,7\% de DCPO)[35]. Weiming et al., buscaron comparar el efecto de la hernioplastía inguinal laparoscópica con técnica transabdominal preperitoneal con y sin fijación de la malla, sobre la incidencia de DCPO 
y la calidad de vida. Contrario a lo descrito, demostraron que realizar este procedimiento sin fijación estaría recomendado en los pacientes con hernia unilateral primaria, quienes presentaron disminución de la frecuencia de DCPO y mejor calidad de vida[36]. Hay una menor frecuencia de DCPO en las mallas más livianas que poseen poros más grandes y por ende menor superficie de contacto, generando una cicatriz más pequeña y preservando la elasticidad del tejido[37].

\section{Cirugía digestiva}

En un estudio retrospectivo, con 199 pacientes sometidos a cirugía colorrectal laparoscópica, la incidencia de DCPO pasados los 2 años desde el procedimiento fue de 17\%. Si bien la intensidad del dolor fue de leve a moderado, lograba un impacto negativo en la calidad de vida del $84 \%$ de los pacientes y produjo alteraciones del sueño en $54 \%$ de ellos. Se identificó como FR para DCPO el DAPQ, enfermedad inflamatoria intestinal y filtración de la anastomosis[38].

\section{Urología}

Artus et al., analizaron a 228 pacientes sometidos a procedimientos urológicos. El $24 \%$ de los pacientes referían DCPO pasados los 3 meses poscirugía. De ellos, 36\% presentaban dolor neuropático. Hubo mayor incidencia de DCPO en pacientes con DAPQ y mayor consumo analgésico en las primeras $48 \mathrm{~h}$ posoperatorias[39].

Alper et al., comparó a 52 pacientes sometidos a nefrectomía abierta versus nefrectomía laparoscópica. No encontró diferencias entre ambos procedimientos en cuanto a intensidad de dolor agudo ni respecto al DCPO. Su incidencia fue de $16 \%$ a los dos meses y $4 \%$ a los seis meses. Sin embargo, los resultados son limitados, debido al pequeño tamaño muestral[40].

Quinientos doce donantes de riñón vivos fueron incluidos y se les aplicó una encuesta de dolor y de calidad de vida. Con respecto a su calidad de vida prevalencia media de DCPO fue del 5,7\%, con un tiempo de seguimiento medio de 6 años. Posibles predictores son dolor posoperatorio temprano intenso, cirugía abdominal previa y lumbago preexistente. El SF - 36 reveló una alteración de la calidad de vida en pacientes con DCPO en comparación con pacientes sin DCPO[41].

\section{Cirugía ginecológica}

El DCPO poshisterectomía tiene una incidencia de 5\%$50 \%$. Esta variabilidad puede ser explicada por diseño de los estudios, definición y población. Han et al., estudiaron un total de 870 pacientes, quienes presentaron una incidencia de DCPO de $27,7 \%$ a los 3 meses. Los predictores independientes de DCPO fueron DAPQ, ansiedad, depresión, insatisfacción sexual y dolor posoperatorio agudo[42].

Yunker et al., estudiaron en 458 pacientes la incidencia de DCPO después de esterilización histeroscópica. En esta serie la incidencia de dolor pélvico agudo fue de $8,1 \%$, mientras que la de dolor persistente después de tres meses, fue de 4,2\%. Aquellas pacientes que previamente padecían de dolor pélvico crónico, dolor lumbar crónico, cefalea o fibromialgia tuvieron seis veces más riesgo de presentar DCPO[43].

\section{Cesárea}

La literatura señala diferentes incidencias de DCPO. Jin et al., describió en 527 mujeres chinas una incidencia de DCPO $18,3 \%, 11,3 \%$, y $6,8 \%$ a los tres, seis y 12 meses respectivamente. La mayoría de las mujeres sufrieron un dolor leve y sólo el 3\% presentó un dolor moderado a severo a los 12 meses. En USA el 2006 madres de habla inglesa refirieron experimentar dolor persistente en relación a la herida quirúrgica en 79\% a los dos meses y $18 \%$ a los seis meses. Weibel et al., en un metaanálisis de 38 artículos describe una incidencia de 11,5\% de dolor persistente a los seis meses y de 11,2\% a los doce meses poscesárea. En su revisión $K$ Sun y $P$ Pan describen un asociación de DCPO con cesáreas previa, mayor consumo de analgesia, depresión preoperatoria, duración de la cirugía, dolor intenso al reposo y movimiento dentro de las primeras $24 \mathrm{~h}$ poscirugía. Se ha teorizado que el estado hormonal del embarazo y el período periparto podrían transmitir cierta protección, probablemente mediada por la oxitocina[44].

\section{Calidad de vida}

Es esperable que un dolor de larga duración interfiera en la vida de los pacientes, limitando su actividad física y disminuyendo su bienestar psicológico[45],[46]. En un estudio realizado por Tolstrup et al., en 440 pacientes sometidos a laparotomía de emergencia, se encontró que aquellos pacientes con DCPO tuvieron un desempeño menor según la escala GIQLI (Gastrointestinal quality of life) en comparación a aquellos sin dolor crónico ${ }^{45}$. Stamer et al., evaluaron cuánto interfiere el DCPO en la vida diaria de 2.400 pacientes sometidos a distintos tipos de cirugía (neurocirugía, cirugía general, ortopédica y ginecológica), utilizando algunas preguntas de la encuesta Brief Pain Inventory. Ellos observaron que a los 6 meses poscirugía, la presencia de DCPO y el dolor de tipo neuropático (según el cuestionario DN4), de forma independiente producen mayor interferencia funcional relacionada al dolor. Los pacientes con dolor neuropático presentaron mayor afectación física y afectiva en comparación con quienes presentaban dolor sin características neuropáticas[46].

\section{Impacto económico}

Existe poca información sobre el impacto económico de este tipo de dolor. Un estudio observacional mostró que $78 \%$ de los pacientes en control médico por dolor postraumático (daño neuronal por trauma) o posquirúrgico ya no tenían trabajo remunerado[47]. La mayoría de quienes se habían invalidado, lo habían hecho por el dolor crónico, mostrando una relación directa entre intensidad del dolor e incapacidad. En pacientes operados de hernia inguinal que evolucionan con DCPO, se ha observado un aumento de costos totales y de costo en prescripciones para el dolor, comparado con los gastos precirugía y con una cohorte control que no presentó DCPO poscirugía[48].

Una posible aproximación al problema podría obtenerse de un estudio realizado para el manejo del dolor lumbar crónico. Este estudio de Hill et al., demostró que los resultados clínicos y de costo-efectividad eran mejores al usar un protocolo basado en las características y FR para cronicidad del paciente, versus un protocolo estándar sólo basado en el diagnóstico[49]. 


\section{Discusión}

EI DCPO es una entidad relativamente nueva, considerando que los primeros artículos científicos respecto del tema fueron publicados a finales de los años ' 90 .

Hemos descrito distintos escenarios quirúrgicos en donde el dolor crónico posoperatorio es prevalente. Los factores de riesgo que se mencionan con mayor frecuencia son el dolor agudo preoperatorio y el dolor agudo posoperatorio. Al parecer la técnica quirúrgica podría ser relevante para una misma patología quirúrgica, sin embargo, los resultados son variables. En ocasiones son contradictorios, mientras algunas publicaciones describen la aparición de factores de riesgo significativos, como la ansiedad preoperatoria, también hay otros que los descartan. Esto ocurre probablemente debido a los diferentes diseños metodológicos y seguimientos observados en los estudios. Está pendiente un acabado conocimiento de los factores de riesgo que pueda determinar para cada paciente una probabilidad de desarrollar DCPO. Eventualmente, la creación de un escala de riesgo permitiría determinar las poblaciones de riesgo en las cuales se pueda realizar intervenciones, tomar medidas preventivas y prolongar los periodos de seguimiento.

Si bien muchos de los pacientes reportan una intensidad de DCPO leve a moderado, quienes lo padecen pueden ver afectada su calidad de vida, su capacidad laboral y requerir mayor atención de salud. Aún desconocemos el real impacto económico de este tipo de dolor. Ejemplo de lo anterior es el dolor crónico poscesárea. A nivel mundial, las cesáreas han ido en aumento llegando el 2015 a ser la vía de parto en aproximadamente $21 \%$ de los nacimientos[50]. Si el DCPO tiene una incidencia reportada que varía entre $1 \%-18 \%$, es muy probable que exista un problema de salud en este ámbito, con un gran impacto en la calidad de vida de las pacientes y que no ha obtenido la atención requerida.

Este estudio, es un estudio de revisión que, pretende actualizar el conocimiento sobre factores de riesgo y prevalencia del DCPO en diferentes escenarios quirúrgicos. Su principal limitación es la de un estudio de revisión narrativa que intenta destacar la disponibilidad de información de dolor crónico posoperatorio sin efectuar un análisis de la calidad de la evidencia ni metaanálisis. Hay que agregar, de acuerdo a lo descrito en la metodología, que cada autor realizó una búsqueda separada de la bibliografía. Aunque se realizó un cruzamiento de las publicaciones seleccionadas y se agregó por consenso aquellas en las que no había más de tres coincidencias, hay espacio para un sesgo de selección dado por el interés de los autores en las áreas quirúrgicas de su especial desempeño.

El conocimiento de la epidemiología e impacto del DCPO en los pacientes es importante para su identificación y oportuno manejo. La creación de herramientas para reconocer a la población de riesgo, que permitan luego instaurar protocolos preventivos, puede contribuir a mejorar la calidad de vida de los pacientes postoperados.

\section{Reconocimientos}

Contribuciones de los autores:

MF BP: Concepción y planificación del estudio original, Selección de artículos, interpretación de los resultados y redacción final del manuscrito.

WA MU: Concepción y planificación del estudio original, Selec- ción de artículos, interpretación de los resultados y redacción final del manuscrito.

MF DG: Búsqueda de artículos, obtención de datos, redacción inicial y final del manuscrito.

MJ FP: Planificación del estudio, selección de artículos, redacción inicial del manuscrito.

JJ OC: Selección de artículos, obtención de datos, redacción inicial del manuscrito.

MA BN: Selección de artículos, obtención de datos, redacción inicial del manuscrito.

OF NV: Selección de artículos, obtención de datos, redacción inicial del manuscrito.

CI CF: Selección de artículos, obtención de datos, redacción inicial del manuscrito.

DTP: Selección de artículos y redacción inicial del manuscrito.

RF CM: Selección de artículos y redacción inicial del documento.

Asistencia para el estudio: ninguna.

Apoyo financiero y patrocinio: ninguno.

Conflictos de interés: ninguno.

Presentaciones: ninguna.

\section{Referencias}

1. Werner MU, Kongsgaard UE. I. Defining persistent post-surgical pain: is an update required? $\mathrm{Br} J$ Anaesth. $2014 \mathrm{Jul} ; 113(1): 1-4$. https://doi.org/10.1093/bja/aeu012 PMID:24554546

2. Macrae WA. Chronic post-surgical pain: 10 years on. Br J Anaesth. 2008 Jul;101(1):77-86. https://doi.org/10.1093/bja/ aen099 PMID:18434337

3. Fletcher D, Stamer UM, Pogatzki-Zahn E, Zaslansky R, Tanase NV, Perruchoud C, et al.; euCPSP group for the Clinical Trial Network group of the European Society of Anaesthesiology. Chronic postsurgical pain in Europe: an observational study. Eur J Anaesthesiol. 2015 Oct;32(10):725-34. https://doi.org/10.1097/ EJA.0000000000000319 PMID:26241763

4. García A, Auad H, Elgueta MF. Prevalencia de Dolor Postoperatorio en Pacientes Sometidas a Mastectomía por Cáncer en el Hospital Clínico de la Universidad Católica, Cohorte 2012. Rev Chil Anest. 2014;43:226-34

5. Hoofwijk DM, Fiddelers AA, Peters ML, Stessel B, Kessels AG, Joosten EA, et al. Prevalence and Predictive Factors of Chronic Postsurgical Pain and Poor Global Recovery 1 Year After Outpatient Surgery. Clin J Pain. 2015 Dec;31(12):1017-25. https://doi. org/10.1097/AJP.0000000000000207 PMID:25565589

6. Han C, Ge Z, Jiang W, Zhao H, Ma T. Incidence and risk factors of chronic pain following hysterectomy among Southern Jiangsu Chinese Women. BMC Anesthesiol. 2017 Aug;17(1):103. https:// doi.org/10.1186/s12871-017-0394-3 PMID:28800726

7. van Rijckevorsel DC, de Vries M, Schreuder LT, Wilder-Smith $\mathrm{OH}$, van Goor H. Risk factors for chronic postsurgical abdominal and pelvic pain. Pain Manag (Lond). 2015;5(2):107-16. https://doi. org/10.2217/pmt.14.47 PMID:25806905

8. Theunissen M, Peters ML, Bruce J, Gramke HF, Marcus MA. Preoperative anxiety and catastrophizing: a systematic review and meta-analysis of the association with chronic postsurgical pain. Clin J Pain. 2012 Nov-Dec;28(9):819-41. https://doi.org/10.1097/ AJP.0b013e31824549d6 PMID:22760489

9. Chatel H, Madar Y, Leyder P, Bonneau C, Barrat C, Quilichini J. 
Prevalence and factors associated with persistent pain following body contouring surgery. J Plast Reconstr Aesthet Surg. 2016 May;69(5):700-5. https://doi.org/10.1016/j.bjps.2016.01.008 PMID:26923660

10. Althaus A, Hinrichs-Rocker A, Chapman R, Arránz Becker $O$, Lefering $R$, Simanski $C$, et al. Development of a risk index for the prediction of chronic post-surgical pain. Eur J Pain. 2012 Jul;16(6):901-10. https://doi.org/10.1002/j.15322149.2011.00090.x PMID:22337572

11. Fregoso G, Wang A, Tseng K, Wang J. Transition from acute to chronic pain: evaluating risk for chronic postsurgical pain. Pain Physician. 2019 Sep;22(5):479-88. PMID:31561647

12. Zaslansky R, Meissner W, Chapman CR. Pain after orthopaedic surgery: differences in patient reported outcomes in the United States vs internationally. An observational study from the PAIN OUT dataset. Br J Anaesth. 2018 Apr;120(4):790-7. https://doi. org/10.1016/j.bja.2017.11.109 PMID:29576119

13. Erlenwein J, Przemeck M, Degenhart A, Budde S, Falla D, Quintel $M$, et al. The Influence of Chronic Pain on Postoperative Pain and Function After Hip Surgery: A Prospective Observational Cohort Study. J Pain. 2016 Feb;17(2):236-47. https://doi.org/10.1016/j. jpain.2015.10.013 PMID:26548971

14. Gungor S, Fields K, Aiyer R, Valle AG, Su EP. Incidence and risk factors for development of persistent postsurgical pain following total knee arthroplasty: A retrospective cohort study. Medicine (Baltimore). 2019 Jul;98(28):e16450. https://doi.org/10.1097/ MD.0000000000016450 PMID:31305475

15. Edgley C, Hogg M, De Silva A, Braat S, Bucknill A, Leslie K. Severe acute pain and persistent post-surgical pain in orthopaedic trauma patients: a cohort study. Br J Anaesth. 2019 Sep;123(3):3509. https://doi.org/10.1016/j.bja.2019.05.030 PMID:31248645

16. Lau, Rick L. et al. Patient Satisfaction after Total Knee and Hip Arthroplasty. Clin Geriatr Med. 28(3): 349 - 365. https://doi. org/10.1016/j.cger.2012.05.001.

17. Thomazeau J, Rouquette A, Martinez V, Rabuel C, Prince N, Laplanche JL, et al. Predictive Factors of Chronic Post-Surgical Pain at 6 Months Following Knee Replacement: Influence of Postoperative Pain Trajectory and Genetics. Pain Physician. 2016 Jul;19(5):E729-41. PMID:27389116

18. Hoofwijk DM, Fiddelers AA, Emans PJ, Joosten EA, Gramke HF, Marcus MA, et al. Prevalence and Predictive Factors of Chronic Postsurgical Pain and Global Surgical Recovery 1 Year After Outpatient Knee Arthroscopy: A Prospective Cohort Study. Medicine (Baltimore). 2015 Nov;94(45):e2017. https://doi.org/10.1097/ MD.0000000000002017 PMID:26559300

19. Wattier JM, Caïazzo R, Andrieu G, Kipnis E, Pattou F, Lebuffe G. Chronic post-thyroidectomy pain: Incidence, typology, and risk factors. Anaesth Crit Care Pain Med. 2016 Jun;35(3):197-201. https://doi.org/10.1016/j.accpm.2015.10.006 PMID:26808897

20. Güven M, Kara A, Yilmaz MS, Demir D, Güven EM. Comparison of Incidence and Severity of Chronic Postsurgical Pain Following Ear Surgery. J Craniofac Surg. 2018 Sep;29(6):e552-5. https:// doi.org/10.1097/SCS.0000000000004532 PMID:29621075

21. Nemati S, Okhovvat SA, Naghavi SE, Shakiba M, Mikaeeli S. Relative frequency of chronic postoperative pain in patients operated for chronic otitis media. Eur Arch Otorhinolaryngol. 2014 Aug;271(8):2139-43. https://doi.org/10.1007/s00405-013-26965 PMID:24052248

22. Bayman EO, Brennan TJ. Incidence and severity of chronic pain at 3 and 6 months after thoracotomy: meta-analysis. J Pain. 2014
Sep;15(9):887-97. https://doi.org/10.1016/j.jpain.2014.06.005 PMID:24968967

23. Niraj G, Kelkar A, Kaushik V, Tang Y, Fleet D, Tait F, et al. Audit of postoperative pain management after open thoracotomy and the incidence of chronic postthoracotomy pain in more than 500 patients at a tertiary center. J Clin Anesth. 2017 Feb;36:174-7. https://doi.org/10.1016/j.jclinane.2016.10.011 PMID:28183561

24. Fiorelli S, Cioffi L, Menna C, Ibrahim M, De Blasi RA, Rendina EA, Rocco M, Massullo D. Chronic Pain After Lung Resection: Risk Factors, Neuropathic Pain, and Quality of Life. J Pain Symptom Manage 2020 Mar 24:S0885-3924(20)30147-0. https://doi. org/10.1016/j.jpainsymman.2020.03.012.. Online ahead of print.

25. Kleiman AM, Sanders DT, Nemergut EC, Huffmyer JL. Chronic Poststernotomy Pain: Incidence, Risk Factors, Treatment, Prevention, and the Anesthesiologist's Role. Reg Anesth Pain Med. 2017 Nov/Dec;42(6):698-708. https://doi.org/10.1097/ AAP.0000000000000663 PMID:28937533

26. Marcassa C, Faggiano P, Greco C, Ambrosetti M, Temporelli PL; Italian Association of Cardiovascular Prevention, Rehabilitation (GICR-IACPR). A retrospective multicenter study on long-term prevalence of chronic pain after cardiac surgery. J Cardiovasc Med (Hagerstown). 2015 Nov; 16(11):768-74. https://doi. org/10.2459/JCM.0000000000000271 PMID:26258718

27. Costa MA, Trentini CA, Schafranski MD, Pipino O, Gomes RZ, Reis ES. Factors Associated With the Development of Chronic Post-Sternotomy Pain: a Case-Control Study. Rev Bras Cir Cardiovasc. 2015 Sep-Oct;30(5):552-6. https://doi.org/10.5935/16789741.20150059 PMID:26735602

28. Urits I, Lavin C, Patel M, Maganty N, Jacobson X, Ngo AL, et al. Chronic Pain Following Cosmetic Breast Surgery: A Comprehensive Review. Pain Ther. 2020 Jun;9(1):71-82. https://doi. org/10.1007/s40122-020-00150-y PMID:31994018

29. Nishimura D, Kosugi S, Onishi Y, Ihara N, Wakaizumi K, Nagata $\mathrm{H}$, et al. Psychological and endocrine factors and pain after mastectomy. Eur J Pain. 2017 Aug;21(7):1144-53. https://doi. org/10.1002/ejp.1014 PMID:28169489

30. Andersen KG, Duriaud HM, Jensen HE, Kroman N, Kehlet $H$. Predictive factors for the development of persistent pain after breast cancer surgery. Pain. 2015 Dec;156(12):2413-22. https:// doi.org/10.1097/j.pain.0000000000000298 PMID:26176893

31. Liot $E$, Bréguet $R$, Piguet $V$, Ris F, Volonté $F$, Morel P. Evaluation of port site hernias, chronic pain and recurrence rates after laparoscopic ventral hernia repair: a monocentric long-term study. Hernia. 2017 Dec;21(6):917-23. https://doi.org/10.1007/s10029017-1663-2 PMID:28864912

32. Takata H, Matsutani T, Hagiwara N, Ueda J, Arai H, Yokoyama $Y$, et al. Assessment of the incidence of chronic pain and discomfort after primary inguinal hernia repair. J Surg Res. 2016 Dec;206(2):391-7. https://doi.org/10.1016/j.jss.2016.08.027 PMID:27884334

33. Andresen K, Burcharth J, Fonnes S, Hupfeld L, Rothman JP, Deigaard $\mathrm{S}$, et al. Chronic pain after inguinal hernia repair with the ONSTEP versus the Lichtenstein technique, results of a doubleblinded multicenter randomized clinical trial. Langenbecks Arch Surg. 2017 Mar;402(2):213-8. https://doi.org/10.1007/s00423016-1532-y PMID:27837273

34. Johansen N, Vyrdal CD, Bisgaard T. Nationwide results on chronic pain after bilateral transabdominal preperitoneal inguinal hernia repair. Scand J Surg. 2019;2019:1-6. https://doi. org/10.1177/1457496919874483 PMID:31514575 
35. Langeveld HR, Klitsie $P$, Smedinga $H$, Eker $H$, Van't Riet M, Weidema W, et al. Prognostic value of age for chronic postoperative inguinal pain. Hernia. 2015 Aug;19(4):549-55. https://doi. org/10.1007/s10029-014-1282-0 PMID:25092408

36. Li W, Sun D, Sun Y, Cen Y, Li S, Xu Q, et al. The effect of transabdominal preperitoneal (TAPP) inguinal hernioplasty on chronic pain and quality of life of patients: mesh fixation versus nonfixation. Surg Endosc. 2017 Oct;31(10):4238-43. https://doi. org/10.1007/s00464-017-5485-1 PMID:28289971

37. Nikkolo C, Vaasna T, Murruste M, Seepter H, Kirsimägi Ü, Lepner U. Randomized clinical study evaluating the impact of mesh pore size on chronic pain after Lichtenstein hernioplasty. J Surg Res. 2014 Oct;191(2):311-7. https://doi.org/10.1016/j. jss.2014.04.022 PMID:24814767

38. Joris JL, Georges MJ, Medjahed K, Ledoux D, Damilot G, Ramquet $C C$, et al. Prevalence, characteristics and risk factors of chronic postsurgical pain after laparoscopic colorectal surgery: retrospective analysis. Eur J Anaesthesiol. 2015 Oct;32(10):712-7. https:// doi.org/10.1097/EJA.0000000000000268 PMID:26086282

39. Artus M, Laviolle B, Maurice A, Malledant Y, Beloeil H. Risk factors for persistent pain after urological surgery. Ann Fr Anesth Reanim. 2014 May;33(5):e89-94. https://doi.org/10.1016/j.annfar.2014.03.013 PMID:24821340

40. Alper I, Yüksel E. Comparison of Acute and Chronic Pain after Open Nephrectomy versus Laparoscopic Nephrectomy: A Prospective Clinical Trial Medicine (Baltimore). 2016; 95(16): e3433. https://doi.org/10.1097/MD.0000000000003433..

41. Bruintjes MH, van Helden EV, de Vries M, Wirken L, Evers AW, van Middendorp $\mathrm{H}$, et al. Chronic pain following laparoscopic living-donor nephrectomy: prevalence and impact on quality of life. Am J Transplant. 2019 Oct;19(10):2825-32. https://doi. org/10.1111/ajt.15350 PMID:30868731

42. Han C, Ge Z, Jiang W, Zhao H, Ma T Incidence and risk factors of chronic pain following hysterectomy among Southern Jiangsu Chinese Women. BMC Anesthesiol 2017 11;17(1): 103. https:// doi.org/10.1186/s12871-017-0394-3.
43. Yunker AC, Ritch JM, Robinson EF, Golish CT. Incidence and risk factors for chronic pelvic pain after hysteroscopic sterilization. J Minim Invasive Gynecol. 2015 Mar-Apr;22(3):390-4. https://doi. org/10.1016/j.jmig.2014.06.007 PMID:24952343

44. Sun KW, Pan PH. Persistent pain after cesarean delivery. Int J Obstet Anesth. 2019 Nov;40:78-90. https://doi.org/10.1016/j. ijoa.2019.06.003 PMID:31281032

45. Tolstrup MB, Thorup T, Gögenur I. Chronic Pain, Quality of Life and Functional Impairment After Emergency Laparotomy. World J Surg. 2019 Jan;43(1):161-8. https://doi.org/10.1007/s00268018-4778-4 PMID:30178128

46. Stamer UM, Ehrler M, Lehmann T, Meissner W, Fletcher D. Pain-related functional interference in patients with chronic neuropathic postsurgical pain: an analysis of registry data. Pain. 2019 Aug;160(8):1856-65. https://doi.org/10.1097/j. pain.0000000000001560 PMID:31335653

47. Parsons B, Schaefer C, Mann R, Sadosky A, Daniel S, Nalamachu $S$, et al. Economic and humanistic burden of post-trauma and post-surgical neuropathic pain among adults in the United States. J Pain Res. 2013 Jun;6:459-69. https://doi.org/10.2147/JPR. S44939 PMID:23825931

48. Elsamadicy AA, Ashraf B, Ren X, Sergesketter AR, Charalambous $L$, Kemeny $H$, et al. Prevalence and cost analysis of chronic pain after hernia repair: a potential alternative approach with neurostimulation. Neuromodulation. Neuromodulation. 2019 Dec;22(8):960-9. https://doi.org/10.1111/ner.12871 PMID:30320933

49. Hill JC, Whitehurst DGT, Lewis M et cols. Comparison of stratified primary care management for low back pain with current best practice (STarT Back): a randomised controlled trial. Lancet 2011 29; 378(9802): 1560-1571. https://doi.org/10.1016/S01406736(11)60937-9..

50. Boerma T, Ronsmans C, Melesse DY, Barros AJ, Barros FC, Juan $\mathrm{L}$, et al. Global epidemiology of use of and disparities in caesarean sections. Lancet. 2018 Oct;392(10155):1341-8. https://doi. org/10.1016/S0140-6736(18)31928-7 PMID:30322584 